\title{
Simulations of magneto-hydrodynamic waves in atmospheres of roAp stars
}

\author{
Elena Khomenko ${ }^{1,2}$ and Oleg Kochukhov ${ }^{3}$ \\ ${ }^{1}$ Instituto de Astrofísica de Canarias, 38205, C/ Vía Láctea, s/n, Tenerife, Spain \\ ${ }^{2}$ Main Astronomical Observatory, NAS, 03680, Kyiv, Ukraine \\ ${ }^{3}$ Department of Physics and Astronomy, Uppsala University, Box 515, SE-751 20, Sweden \\ email: khomenko@iac.es
}

\begin{abstract}
We report 2D time-dependent non-linear magneto-hydrodynamical simulations of waves in the atmospheres of roAp stars. We explore a grid of simulations in a wide parameter space. The aim of our study is to understand the influence of the atmosphere and the magnetic field on the propagation and reflection properties of magneto-acoustic waves, formation of shocks and node layers.
\end{abstract}

Keywords. MHD - stars: magnetic fields - stars: oscillations

RoAp stars are late-A, chemically peculiar stars with effective temperatures between 6500-8100 K and global dipolar-like magnetic fields with strengths between 1-25 kG. They show low-order angular degree $p$-mode pulsations with periods between 4 and 22 minutes. These non-radial pulsations are aligned with dipolar field axis. Magneto-acoustic oscillations in peculiar A stars are of particular interest due to unique opportunities to study the interaction of pulsations, chemical inhomogeneities, and strong magnetic fields. Recent reviews on the properties of these stars and their pulsations can be found in Kurtz (2008); Cunha (2007); Kochukhov (2007, 2008).

We solve the basic 2D non-linear adiabatic equations of the ideal MHD by means of the numerical code described by Khomenko \& Collados (2006); Khomenko et al. (2008). We assume that: (1) the magnetic field varies on spatial scales much larger than the typical wavelength, allowing the problem to be solved locally for a plane-parallel atmosphere with a homogeneous inclined magnetic field; (2) waves in the atmosphere are excited by low-degree pulsation modes with radial velocities exceeding horizontal velocities. The unperturbed atmospheric model has an effective temperature of $T_{\text {eff }}=7750 \mathrm{~K}$ and gravitational acceleration at the surface $\log _{10} g=4.0$. The simulation grid covers the magnetic field strength $B=1-7 \mathrm{kG}$; magnetic field inclination to the local vertical $\gamma=0-60$ degrees and driving periods $T=6-13$ minutes. An example of the wave pattern developed in the simulations is given in Fig. 1. We use the longitudinal and transversal field projections of the velocity in order to separate clearly the fast and slow MHD waves. Our first results pick up some observed properties of the roAp stars pulsations, such as: rapid growth of the wave amplitude with height, presence of the node surfaces, large variations in pulsation properties depending on the parameters of the model. Velocity signal observed in the upper atmospheric layers of roAp stars is mostly due to running slow mode (acoustic) waves propagating along the inclined magnetic field lines. The node structures and the rapid phase variations at the lower atmospheric layers are due to multiple reflections and interference of the slow and fast mode waves. The disc-integrated velocity signal produced by the atmospheric pulsations of such a star will depend in a complex way on the inclination of the magnetic axis with respect to the observational line of sight and will be a subject of our further study. 

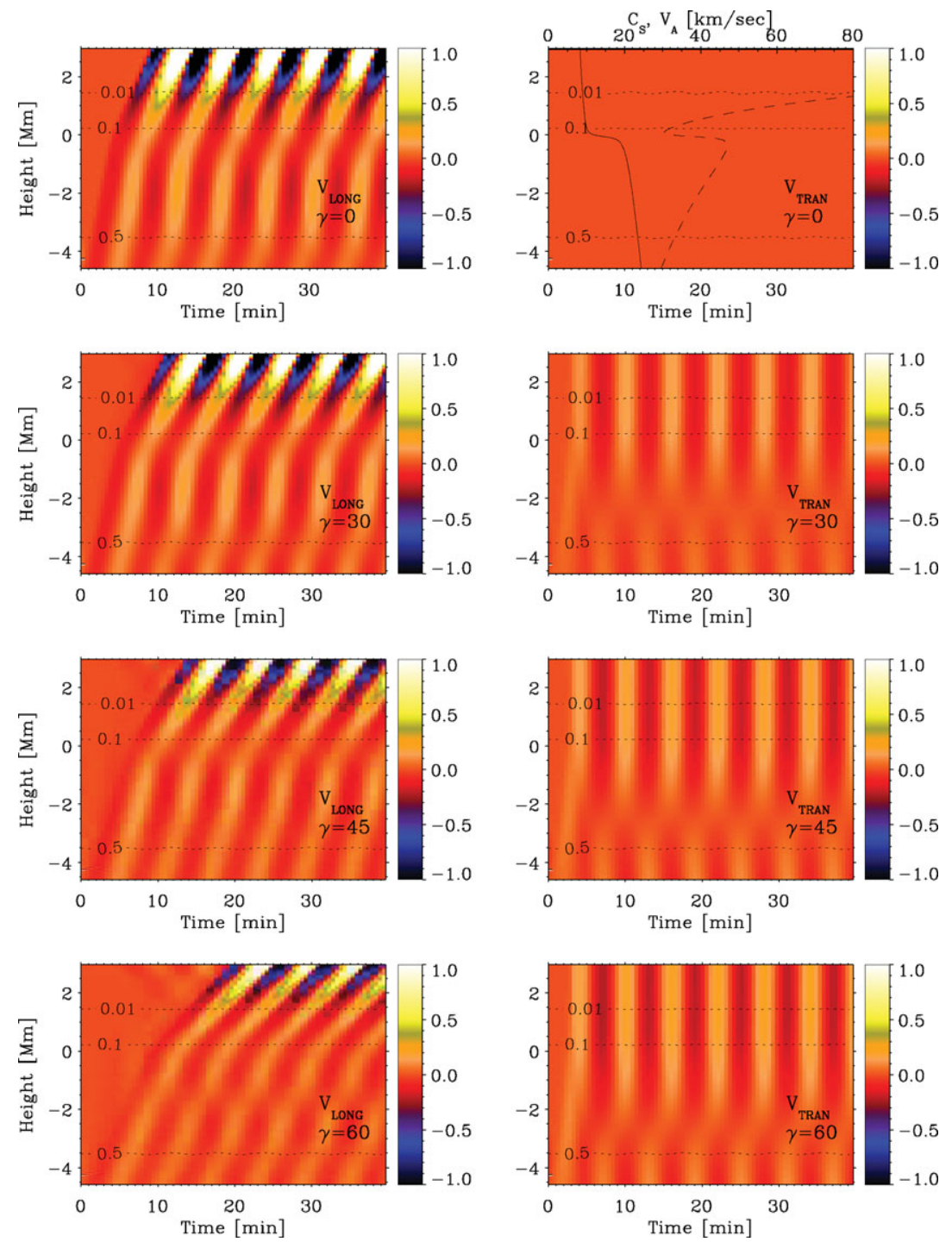

Figure 1. Height-time variations of the longitudinal (parallel to the field, left) and transversal (perpendicular to the field, right) velocities for $\mathrm{B}=1 \mathrm{kG}, \mathrm{T}=360 \mathrm{sec}$ at latitudes where the inclination equals $0,30,45$ and 60 degrees. The color bars give velocity scale in $\mathrm{km} / \mathrm{sec}$. Zero height corresponds to the photospheric base. Dotted lines marked with numbers are contours of constant $c_{S}^{2} / v_{A}^{2}$. Height dependences of the sound speed $c_{S}$ (solid line) and Alfvén speed $v_{A}$ (dashed line) are plotted over the top panel, the scale is given by the upper axis.

\section{References}

Cunha, M. S. 2007, Comm. in Astroseismology 150, 48

Khomenko, E. \& Collados, M. 2006, ApJ 653, 739

Khomenko, E., Collados, M., \& Feliipe, T. 2008, SP 251, 589

Kochukhov, O. 2007, Comm. in Astroseismology 150, 39

Kochukhov, O. 2008, Comm. in Astroseismology arXiv:0810.1508, in press

Kurtz, D. W. 2008, SP 251, 21 